\title{
Análisis de dominios científicos nacionales en Comunicación (Scopus, 2003-2018)
}

\section{Analysis of national scientific domains in the Communication field (Scopus, 2003-2018)}

Javier Trabadela-Robles; María-Victoria Nuño-Moral; Vicente P. Guerrero-Bote; Félix De-Moya-Anegón

Cómo citar este artículo:

Trabadela-Robles, Javier; Nuño-Moral, María-Victoria; Guerrero-Bote, Vicente P.; De-Moya-Anegón, Félix (2020). "Análisis de dominios científicos nacionales en Comunicación (Scopus, 2003-2018)". Profesional de la información, v. 29, n. 4, e290418.

https://doi.org/10.3145/epi.2020.jul.18

Artículo recibido el 21-02-2020 Aceptación definitiva: 05-05-2020
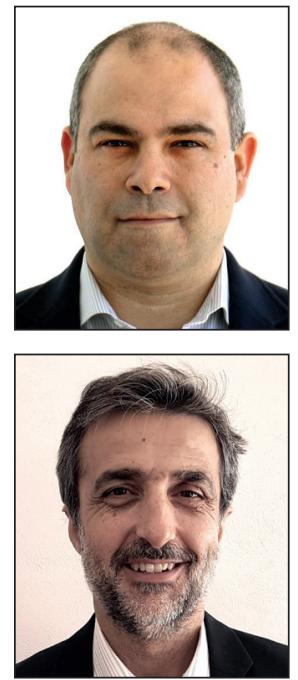

Javier Trabadela-Robles $\triangle$ https://orcid.org/0000-0001-5338-9257

Universidad de Extremadura Fac. de Ciencias de la Doc. y la Com. Dpto. de Información y Comunicación Plazuela Ibn Marwam, s/n. 06071 Badajoz, España jtrarob@unex.es

Vicente P. Guerrero-Bote http://orcid.org/0000-0003-4821-9768

Universidad de Extremadura Fac. de Ciencias de la Doc. y la Com. Dpto. de Información y Comunicación Plazuela Ibn Marwam, s/n. 06071 Badajoz, España guerrero@unex.es

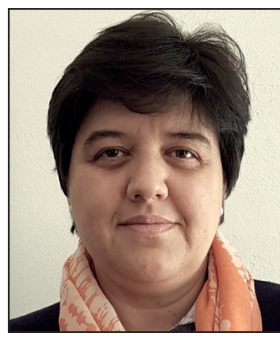

María-Victoria Nuño-Moral https://orcid.org/0000-0002-5447-4813

Universidad de Extremadura

Fac. de Ciencias de la Doc. y la Com. Dpto. de Información y Comunicación Plazuela Ibn Marwam, s/n. 06071 Badajoz, España mvnunmor@unex.es

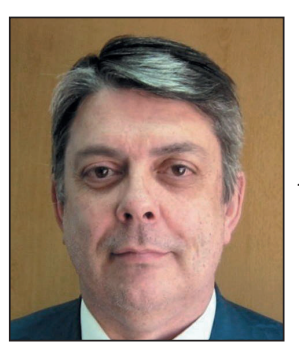

Félix De-Moya-Anegón https://orcid.org/0000-0002-0255-8628

SCImago Research Group, España felix.moya@scimago.es

\section{Resumen}

En los últimos años la disciplina Comunicación ha experimentado un gran crecimiento en investigación. Los recientes cambios tecnológicos, así como la irrupción de la web 2.0 y 3.0 y nuevas formas de comunicación, como las redes sociales, han propiciado nuevos y variados estudios. En este trabajo se analiza la producción científica de los 27 países con mayor producción científica en Comunicación en el período 2003-2018. Algunos resultados son: los países más productivos son Estados Unidos, Reino Unido y España; y hay menos colaboración internacional que en otras disciplinas, sin embargo, existe una red de colaboración europea bastante densa y de gran impacto, y otra red de menor densidad e impacto de países del Pacífico (Asia + Oceanía).

\section{Palabras clave}

Comunicación; Cienciometría; Evaluación de la investigación; Comunicación académica; Colaboración internacional; Redes de colaboración; Excelencia científica; Scopus.

\section{Agradecimientos}

Investigación finaciada por la Junta de Extremadura (Consejería de Educación, Ciencia y Tecnología) y por la Unión europea "Fondo Europeo de Desarrollo Regional. Una manera de hacer Europa" como parte de las ayudas a los grupos GR18042 y GR18044. 


\begin{abstract}
Scientific research in the communication field has experienced great growth in recent years. Recent technological changes, as well as the emergence of Web 2.0 and 3.0 and new forms of communication, such as social networks, have led to new and varied studies in the discipline. This paper analyzes the scientific production of the 27 countries with the greatest scientific production in the communication field in the period from 2003 to 2018 . The results indicate that: the most productive countries are the USA, UK, and Spain; there is less international collaboration than in other disciplines, but there is a network of European collaboration that is quite dense and has great impact, and another network in Pacific countries (Asia + Oceania), albeit with lower density and impact.
\end{abstract}

\title{
Keywords
}

Communication; Scientometrics; Research evaluation; Scholarly evaluation; Scholarly communication; Assessment; International collaboration; Collaboration networks; Scientific excellence; Scopus.

\section{Introducción}

Comunicación es un concepto amplio. Incluso si nos vamos a la ciencia y la tecnología podemos encontrar las tecnologías de la comunicación, la comunicación académica, la comunicación de la ciencia, etc. Pero, lo que en las Ciencias Sociales se identifica como disciplina, como ciencia de la Comunicación, incluye el estudio de la comunicación de masas, el periodismo, la televisión, la radio o la publicidad y actualmente, también incluye los medios o redes sociales.

Comunicación está presente en las grandes bases de datos bibliográficas de documentación científica como categoría o área independiente, y por su reciente desarrollo tiene interés el análisis de la producción científica en Comunicación de los principales países, así como de la colaboración entre los mismos. Revisando la bibliografía científica no encontramos ningún análisis previo de ese tipo, aunque sí encontramos otros estudios bibliométricos sobre patrones de publicación, la red de citación, etc.:

Lauf (2005) analiza los patrones de publicación de las revistas más importantes de Comunicación entre 1998 y 2002 mostrando que hay una sobrerrepresentación de revistas y autores estadounidenses, seguidas por las de otros países angloparlantes. Demeter (2018) repite el estudio concluyendo que continúa el dominio, no tanto estadounidense sino de los países desarrollados. Méndez-Rátiva y Gregorio-Chaviano (2014) hicieron una aproximación desde la bibliometría: analizaron los trabajos presentados en las revistas del primer cuartil (Q1) de los Journal Citation Reports (JCR) en la categoría de Comunicación de 2008 a 2011 evidenciando la predominancia de los trabajos estadounidenses y como temas la comunicación, la información y los medios de comunicación.

Kolle, Rabindranath y Shankarappa (2019) hacen un estudio bibliométrico de los artículos más citados (más de 100 citas) de Comunicación en la Web of Science (WoS) (1989-2018), analizando también la red de colaboración de países. En ella, Estados Unidos tiene el $71 \%$ de los documentos, seguidos del Reino Unido con el 9\%, jugando así el mundo inglés el papel central en la red de colaboración.

Park y Leydesdorff (2009) analizaron la matriz de citación de revistas de Comunicación tomando como punto de partida el Journal of communication, utilizando los datos de los JCR de 2006. Encuentran que las revistas de psicología social y experimental son las principales fuentes. También indican que las revistas de publicidad y de los nuevos medios no están muy integradas en el núcleo dominado por revistas estadounidenses. Leydesdorff y Probst (2009) también utilizaron la citación entre revistas extraídas de los JCR para relacionarlas con las de psicología social y política llegando a la conclusión de que la disciplina de Comunicación se está desarrollando progresivamente.

Barnett et al. (2011) analizaron la red de citación de la categoría Communication de la WoS en 2009 y hallaron que la estructura de influencia de la misma es más compleja que la que encontraron Park y Leydesdorff (2009). Y Montero-Díaz et al. (2018), que analizaron la evolución de la estructura conceptual de la categoría Communication de la WoS desde 1980 a 2013, concluyeron que está bastante fragmentada, encontrando dieciséis temas de interés, lejos de los dos bloques que encontraron Barnett et al. (2011) (comunicación de masas e interpersonal).

Sin embargo, donde hay más estudios bibliométricos de Comunicación es a nivel español y latinoamericano. En 2006, Castillo-Esparcia y Xifra (2006) analizaron la investigación doctoral en Relaciones Públicas en España de 1965 a 2005 concluyendo que la mayoría se centraban en aspectos profesionales, aunque una parte también trataba la dimensión informativa. Repiso, Torres-Salinas y Delgado-López-Cózar (2011a; 2011b) estudiaron las tesis doctorales en radio y en televisión defendidas en las universidades españolas de 1976 a 2008 hallando un crecimiento constante y un papel relevante de las universidades históricas en Comunicación.

Castillo-Esparcia y Carretón-Ballester (2010) llevaron a cabo un estudio bibliométrico de las diez revistas españolas de Comunicación de mayor impacto de acuerdo con el índice InRecs, observando que los principales temas de investigación son por este orden: periodismo, responsabilidad social en los medios, televisión, publicidad, internet y las nuevas tecnologías. Castillo-Esparcia, Rubio-Moraga y Almansa-Martínez (2012) hicieron un análisis bibliométrico de las revistas de 
mayor impacto de los JCR en el año 2008, considerando la procedencia, colaboración y género de los autores, junto con los principales temas. Álvarez-Nobell y Castillo-Esparcia (2015) deconstruyen las perspectivas y enfoques teóricos de los autores de las revistas latinoamericanas de Comunicación incluidas en Scopus a lo largo de quince años, sobresaliendo las escasas referencias a autores clásicos.

Fernández-Quijada, Masip y Bergillos (2013) examinaron los patrones de autoría de los trabajos científicos en comunicación publicados por autores españoles, encontrando dos patrones dependiendo de si los trabajos se publicaban en revistas nacionales o internacionales. Fernández-Quijada y Masip (2013) revisaron la evolución de la investigación española en Comunicación de 1980 a 2010, tanto a nivel nacional como internacional, encontrando la misma dualidad. De-las-Heras-Pedrosa, Martel-Casado y Jambrino-Maldonado (2018) hicieron un estudio bibliométrico de los autores españoles de Comunicación (2016-2018) en Scopus. Observaron que eran determinantes las exigencias de la Aneca y la Cneai en el crecimiento de la producción, así como que la investigación se va centrando en las redes sociales, la comunicación política, la universidad o los estudios de género.

De-Filippo (2013) analizó la producción científica española en Communication de los JCR en el lustro 2007-2012, comparándola con la del resto del mundo. España ocupaba la cuarta posición como editora de revistas y la sexta como productora de artículos. Sugirió que había margen de mejora en la producción científica nacional, en la inclusión de nuevas revistas en los JCR y en la internacionalización, tanto por la colaboración internacional como por la inclusión de trabajos españoles en revistas internacionales. Baladrón-Pazos, Manchado-Pérez y Correyero-Ruiz (2017) hicieron un estudio bibliométrico de la producción científica sobre Publicidad en España (1980-2015) con una muestra de 1.050 artículos de 80 revistas españolas de Comunicación. Sus resultados fueron: escasa internacionalización, bajo nivel de colaboración, y ausencia de una red cohesionada. También encontraron que más del 35\% de los trabajos estaban dedicados a la creatividad y al mensaje publicitario. Rodríguez-Gómez, Goyanes y Rosique-Cedillo (2018) entre otras cosas, examinaron la producción científica publicada en las 11 revistas españolas de mayor impacto en Comunicación analizando el número, los métodos y los temas.

Moreno-Delgado, Repiso y Montero-Díaz (2020) llevaron a cabo un análisis bibliométrico y de redes sociales de la producción científica sobre programación televisiva incluida en la WoS encontrando que existen dos aspectos: la programación propiamente dicha y su influencia en otras dimensiones.

Nuestro objetivo en este trabajo, como ya hemos avanzado previamente, es hacer un amplio estudio de la producción científica de los principales países en Comunicación prestando especial atención a la colaboración. Todo ello con el fin de responder a preguntas como:

- ¿Cómo evoluciona la producción mundial en Comunicación en el período 2003-2018?

- ¿Cómo es la producción científica en Comunicación de los principales países, su impacto científico y la colaboración entre los mismos?

- ¿Existe relación entre el impacto científico y la colaboración internacional?

- ¿Cuáles son las redes nacionales de colaboración científica en Comunicación más densas?

\section{Datos y método}

Los datos han sido extraídos de SCImago Journal \& Country Rank (SJ\&CR) y de SCImago Institutions Rankings (SIR), plataformas creadas por el grupo SCImago basadas en la información incluida en la base de datos Scopus (SCImago, 2020a; 2020b). La base de datos Scopus de Elsevier (Hanne, 2004; Pickering, 2004) es una de las bases de datos bibliográficas que incluye un número mayor de revistas y congresos científicos. A pesar de llevar un tiempo no muy largo ofreciendo sus servicios, ha sido objeto de estudio y análisis en diversas investigaciones (Archambault et al., 2009; Leydesdorff; De-Moya-Anegón; Guerrero-Bote, 2010; De-Moya-Anegón et al., 2007), y ha sido utilizada en muchos estudios cienciométricos (Gorraiz; Gumpenberger; Wieland, 2011; Jacsó, 2011; Corera-Álvarez; De-Moya-Anegón; 2009; Romo-Fernández et al., 2011; Guerrero-Bote; De-Moya-Anegón, 2015).

En Scopus, SJ\&CR y SIR, los documentos y las revistas/congresos están clasificados por Áreas Temáticas y por Áreas Temáticas Específicas o Categorías. Hay más de trescientas Áreas Temáticas Específicas que están agrupadas en veintiséis Áreas Temáticas. Además, está el Área Temática Multidisciplinar que contiene revistas multidisciplinares como Nature o Science.

Una particularidad de Scopus es que al incluir congresos, particularizando a determinadas disciplinas, pueden verse variaciones anuales en el número de documentos debido a que algunos congresos tienen una periodicidad inferior a la anual.

Dentro del Área Temática Social Sciences, que se compone de veintitrés Áreas Temáticas Específicas (más una miscelánea de las Ciencias Sociales), se encuentra la categoría Communication. El número de revistas/congresos científicos incluidos en Communication parte de 117 en 2003, y llega a 411 en 2018 (3,5 veces más). En WoS/JCR también existe una categoría científica Communication que parte de 44 revistas en 2003, y se duplica en 2018 con 88 . Se pone de manifiesto que Scopus no sólo incluye más fuentes, sino que su número crece más durante el período. 
Los datos extraídos para este trabajo corresponden a los 27 países más productivos dentro de la categoría de Communication de Scopus en el período 2003-2018 (en el momento de realizar el trabajo no se disponen todavía de datos completos de 2019). Cada uno de esos países ha publicado más de novecientos documentos en estos 16 años, y entre todos acumulan 105.094 documentos, que son casi el 92\% de la producción mundial recogida en esta base de datos. Veintisiete países, acumulando el $92 \%$ de la producción mundial, nos parece un número representativo para tener una visión mundial, así como suficientemente manejable para un estudio como éste.

Los indicadores que hemos utilizado para caracterizar la producción científica en Comunicación son los siguientes:

- Ndoc: número de documentos publicados en revistas científicas recogidas en la base de datos de Scopus.

- \%Ndoc: porcentaje que suponen los documentos de Comunicación con respecto al total de la producción global o del país en cuestión.

- Citas por documento: promedio de citas por documento. La citación depende en gran medida del tiempo que el documento haya tenido para ser citado, por esta razón no se evalúa la evolución de este indicador.

- \% documentos citados: al igual que el indicador anterior depende en gran medida del tiempo que los documentos han tenido para ser citados, por esta razón no se evalúa su evolución.

- \% colaboración internacional: porcentaje de los documentos realizados por autores de varios países.

- Liderazgo y \% Liderazgo: número y porcentaje de documentos publicados en revistas científicas recogidas en Scopus en los que un autor del correspondiente país ha actuado como líder (autor de correspondencia) (De-Moya-Anegón et al., 2013).

- Impacto normalizado (IN): promedio de la citación normalizada recibida por cada documento, entendiéndose ésta como la ratio entre la citación recibida por el documento y la citación promedio de los documentos del mismo tipo, año y categoría (Rehn; Kronman, 2008).

- Excelencia10 y \% Excelencia10: número y porcentaje de documentos que están en el 10\% más citado del mismo año, tipo y categoría (Bornmann; De-Moya-Anegón; Leydesdorff, 2012).

- Excelencia10 liderada y \% excelencia10 liderada: Número y porcentaje de documentos que están en el 10\% más citado del mismo año, tipo y categoría en los que un autor del correspondiente país ha actuado como líder (autor de correspondencia).

- Excelencia1 y \% Excelencia1: número y porcentaje de documentos que están en el 1\% más citado del mismo año, tipo y categoría.

- Tasa de variación (TV): para ver la evolución de los indicadores anteriores en este período se ha calculado una tasa de variación entre el promedio del primer trienio (2003-2005) y el promedio del trienio 2015-2017; se ha evitado utilizar el año 2018 porque la citación de este año no se puede considerar estable en el momento en el que se descargaron los datos.

En este trabajo todos estos indicadores se han aplicado a la producción científica en el área de Comunicación. Es decir, cuando hablamos del Impacto Normalizado de un país nos referimos al Impacto Normalizado de su producción científica en Comunicación, cuando hablamos de la Excelencia10 liderada de un país nos referimos a su Excelencia10 liderada en Comunicación, y así sucesivamente.

Con los datos de todos estos indicadores se ofrece una descripción bibliométrica detallada de la producción de estos 27 países en Comunicación. Sin embargo, con el fin de tener una visión más general del comportamiento de cada país se ha realizado un escalamiento multidimensional. Para realizar ese mapa, en primer lugar se han normalizado los valores de cada indicador y de sus tasas de variación por el máximo valor de cada indicador; después se han reducido al $10 \%$ los valores de los indicadores dependientes del tamaño, para evitar que focalicen la clasificación. Seguidamente, se ha realizado una normalización euclídea de cada vector compuesto por 26 componentes, con el fin, de eliminar las diferencias en módulo y clasificar cada país en función de los valores relativos de cada indicador. Y finalmente, se ha calculado la distancia euclídea entre cada pareja de países, que se ha utilizado para realizar el escalamiento multidimensional (alscal).

La producción científica en Comunicación ha ido aumentando desde 2003 a un ritmo de un $23 \%$ anual

\section{Resultados}

En el período de estudio, la producción en Comunicación en el mundo ha tenido un crecimiento sostenido, pasando de 3.105 documentos en 2003 a 14.575 en 2018 (figura 1), lo que supone un crecimiento de un 369\%; es decir, la producción ha ido aumentando a un ritmo de un $23 \%$ anual de media. Como se ha indicado anteriormente, durante este período se ha incrementado el número de revistas de Comunicación un $251 \%$. Por una parte ello se debe al incremento de cobertura de Scopus en todas las materias, pero también es consecuencia del incremento de producción y madurez de la investigación en Comunicación.

Si se compara la producción en Comunicación con el resto de producción mundial, así como su evolución en el período de estudio (figura 1), se aprecia que crece desde un $0,20 \%$ hasta un $0,47 \%$. Esto supone un aumento con respecto a la 
producción mundial de un $136 \%$, es decir, casi de un $8,5 \%$ de crecimiento anual medio. Como se puede ver, este crecimiento no ha sido monótono, sino que ha habido años en los que ha disminuido, como en 2004 y en 2017. Esos vaivenes se deben a congresos que existieron los años previos (2003 y 2016, y en menor medida 2007 y 2011) que no han tenido continuidad en los años sucesivos. Por todo ello, podemos concluir que ha sido una disciplina que ha crecido considerablemente en esta última década y media. Además, se puede apreciar que la producción en Comunicación ha crecido a un ritmo bastante superior (bastante más del triple) al de la producción mundial total.

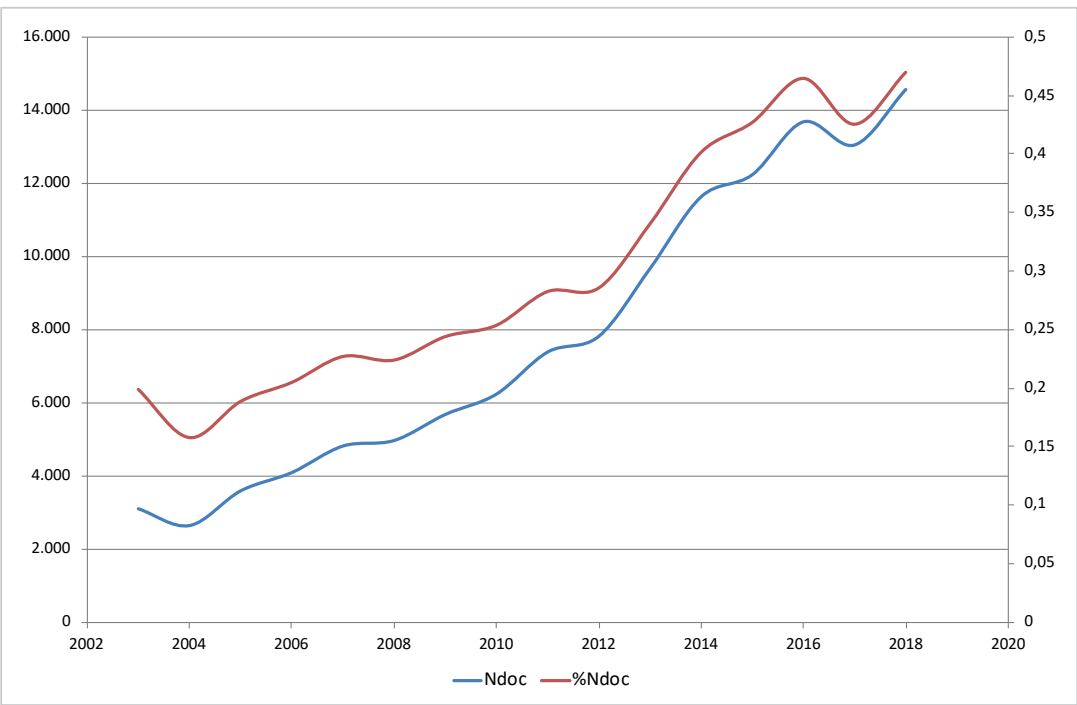

Figura 1. Producción de Comunicación en el período 2003-2018, tanto en número de papers como en porcentaje con respecto a la producción científica total mundial.

En la figura 2 se puede observar una gran correlación entre el Impacto normalizado y el \% Excelencia liderada de los 27 países más productivos $\left(R^{2}=0,80\right)$ para Comunicación. También podemos ver en los círculos concéntricos las Ndocs de los países, respectivamente Ndoc, Liderazgo, Excelencia10 y Excelencia10 liderada. En esta área científica no hay mucha colaboración internacional, por eso es muy poca la diferencia entre Ndoc y Liderazgo por una parte y entre Excelencia10 y Excelencia10 liderada. Para apreciar esos datos se han elaborado las tablas 1 y 2 . En ellas se puede ver como hay países como India o Estados Unidos donde prácticamente no se aprecian diferencias entre Ndocs y Liderazgo, y otros países como Suiza o Singapur con mayores diferencias. La Excelencia10 ocupa una gran parte de la producción en algunos países como Países Bajos, Noruega o Suiza frente a otros en el extremo contrario como India, Malasia, China y Sudáfrica.

Si se observa el indicador de Ndoc, se puede apreciar que hay un país, Estados Unidos, muy por encima del resto en trabajos publicados, con 43.730 (más de un tercio de la producción del total de los 27 países objeto de estudio). Le sigue Reino Unido con casi 12.000 publicaciones y España con 6.533. Entre los tres países producen más de la mitad del total de los 27 países.

Si se analiza la tasa de variación (TV), se observa que todos los países estudiados han crecido, siendo los que más lo han hecho: Malasia, Brasil, India, China y España. Aunque también crecieron, lo hicieron en menor medida en comparación con el resto: Nueva Zelanda, Estados Unidos y Reino Unido. Como hemos dicho, en estos dos últimos países se da la mayor producción en Comunicación, pero no aumenta tanto proporcionalmente en comparación con los otros países del estudio.

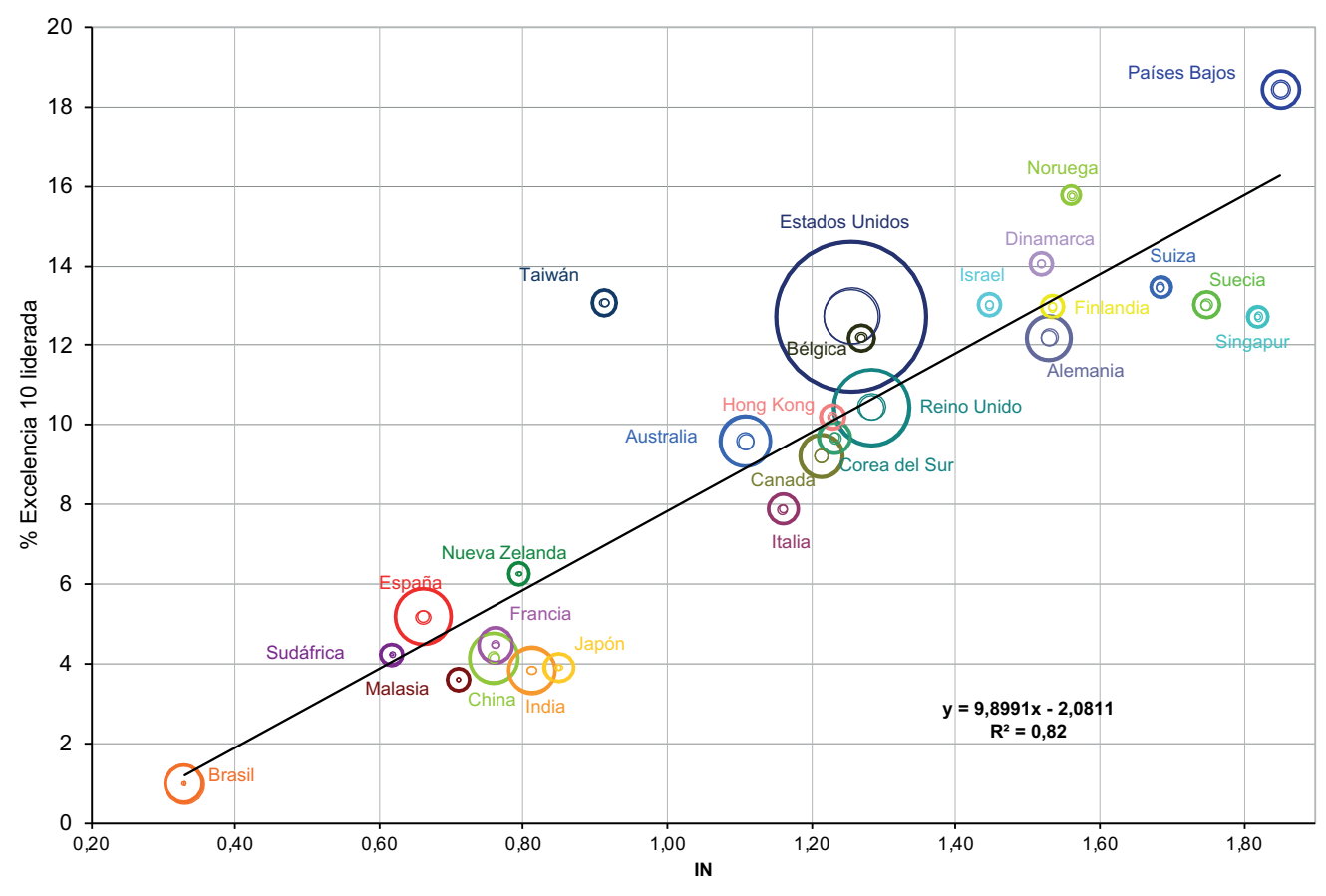

Figura 2. Impacto normalizado respecto a \%Excelencia10 liderada de los 27 países con mayor producción. Los círculos respresentan respectivamente: Ndoc, Liderazgo, Excelencia10 y Excelencia10 liderada. Todo ello en Comunicación. 
Mirando el esfuerzo que hace cada país en el indicador de \%Ndoc, que es el porcentaje del total de la producción científica de cada país categorizada como Comunicación, vemos otro panorama diferente teniendo en mente que el promedio mundial es del 0,32\%. La media de este indicador en los 27 países estudiados es de $0,33 \%$, por lo que se observa que en los países de mayor producción, ésta tiene un peso específico ligeramente superior a la media en esta disciplina en comparación a la producción científica general. Destacan España y Nueva Zelanda con un 0,56\%. Sin embargo, en Japón y China esta producción específica tan sólo representa el 0,09\% del total de sus trabajos publicados. España también tiene una alta tasa de variación, situándose en este aspecto la segunda, tras Brasil y por delante de India, Suiza y China.

En lo que se refiere a las citas de los trabajos, no siempre ocurre que los países que más producen son los más citados. El país con el mayor promedio de citas por documento es Holanda (décimo país en producción), seguido de Estados Unidos (1ㅇe en producción), Israel (20으), Singapur (26으) y Taiwán (17으). Si se observa el porcentaje de documentos producidos que son citados (\% Documentos citados), en primer lugar estaría Singapur, seguido de Holanda, Israel, Finlandia, Noruega y Suecia (todos ellos en torno al $75 \%$ de promedio de citación de trabajos). Los países menos citados porcentualmente son Brasil, China e India (entre el 43 y el 49\%), probablemente porque son de los que más crecen en producción en el período estudiado (por lo que la producción es más reciente y, por tanto, ha tenido menos posibilidades de ser citada).

Respecto a la colaboración internacional, los que más producen con otros países son Singapur y Suiza con casi el $47 \%$, mientras que, en el lado opuesto, están India (7\%), junto con Estados Unidos y Brasil con un 13\%. Hay tasas de variación negativas para Corea del Sur, India, Malasia y Taiwán. Llama la atención que de los países que más han crecido en producción están Malasia, Brasil e India y, atendiendo a los dos indicadores anteriores de la colaboración internacional, hace suponer que este gran crecimiento no se ha producido por el establecimiento de lazos internacionales sino, al contrario, por la producción sin colaboración. De hecho, si miramos la correlación del \% de colaboración internacional con el resto de indicadores, observamos una correlación por encima de 0,5 con los indicadores de rendimiento independientes del tamaño. Y tiene una correlación negativa tanto con el porcentaje de liderazgo (lo cual puede resultar lógico) y de cerca de -0.2 con el \%Ndoc. Esto último puede sugerir que esos países que han aumentado tanto su producción lo han hecho con producción propia sin colaboración internacional, quizá debido a la incorporación de revistas nacionales a la base de datos, que incluyen trabajos de menor calidad y sin colaboración.

El liderazgo es el indicador que mayor correlación tiene con el Ndoc, correlación que se extiende a sus tasas de variación. El porcentaje de liderazgo no tiene grandes correlaciones con otros indicadores, salvo la negativa con la colaboración

Tabla 1. Ndoc, \%Ndoc, Citas por documento, \% Documentos citados, \% Colaboración internacional, Liderazgo y \%Liderazgo, de los 27 países más productivos y las tasas de variación del trienio 2015-2017 con respecto al trienio 2003-2005.

\begin{tabular}{|c|c|c|c|c|c|c|c|c|c|c|c|c|}
\hline País & Ndoc & TV & \%Ndoc & TV & $\begin{array}{c}\text { Citas por } \\
\text { documento }\end{array}$ & $\begin{array}{c}\text { \% Documentos } \\
\text { citados }\end{array}$ & $\begin{array}{c}\text { \% Colaboración } \\
\text { internacional }\end{array}$ & TV & Liderazgo & TV & $\begin{array}{c}\% \\
\text { Liderazgo }\end{array}$ & $\mathrm{TV}$ \\
\hline Estados Unidos & 43730 & 170,90 & 0,46 & 90,69 & 11,40 & 72,10 & 13,36 & 92,17 & 40763 & 157,92 & 93,22 & $-4,79$ \\
\hline Reino Unido & 11928 & 228,06 & 0,43 & 101,70 & 9,52 & 67,97 & 22,63 & 85,96 & 10617 & 199,14 & 89,01 & $-8,82$ \\
\hline España & 6533 & 1985,15 & 0,56 & 898,65 & 4,39 & 57,75 & 18,83 & 10,52 & 5909 & 1943,33 & 90,45 & 2,01 \\
\hline Australia & 5251 & 449,50 & 0,45 & 129,88 & 7,74 & 70,27 & 23,12 & $-3,02$ & 4678 & 462,69 & 89,09 & 2,40 \\
\hline China & 5119 & 1991,89 & 0,09 & 418,53 & 2,85 & 47,22 & 27,23 & $-3,90$ & 4635 & 2105,15 & 90,55 & 5,41 \\
\hline India & 4514 & 2477,48 & 0,30 & 537,24 & 2,14 & 49,49 & 7,29 & $-44,63$ & 4345 & 2522,64 & 96,26 & 1,75 \\
\hline Alemania & 4071 & 583,03 & 0,17 & 328,99 & 8,71 & 66,52 & 35,81 & 25,36 & 3404 & $\square 530,65$ & 83,62 & $-7,67$ \\
\hline Canadá & 3884 & 345,32 & 0,27 & $\square 164,51$ & 8,83 & 68,36 & 29,09 & 10,84 & 3253 & 341,56 & 83,75 & $-0,85$ \\
\hline Brasil & 3253 & 3253,13 & 0,39 & 1002,34 & 1,89 & 43,31 & 13,86 & 13,33 & 3090 & 3372,41 & 94,99 & 3,56 \\
\hline Países Bajos & 3184 & 239,69 & 0,40 & 90,02 & 14,01 & 77,42 & 37,66 & 65,69 & 2627 & 187,50 & 82,51 & $-15,37$ \\
\hline Francia & 2705 & 278,54 & 0,16 & 142,53 & 4,56 & 52,27 & 31,13 & 157,96 & 2240 & 214,78 & 82,81 & $-16,84$ \\
\hline Corea del Sur & 2286 & 805,00 & 0,23 & 258,83 & 8,12 & 66,45 & 34,60 & $-47,21$ & 1927 & 941,56 & 84,30 & 15,09 \\
\hline Italia & 2105 & 813,79 & 0,15 & 375,78 & 6,22 & 58,15 & 32,78 & 3,96 & 1769 & 730,38 & 84,04 & $-9,13$ \\
\hline Japón & 1896 & 394,35 & 0,09 & 355,41 & 5,86 & 58,60 & 4,42 & 4,30 & 644 & 400,00 & 86,71 & 1,14 \\
\hline Suecia & 1706 & 660,76 & 0,33 & 328,22 & 9,98 & 74,44 & 31,71 & 97,17 & 1416 & 545,21 & 83,00 & $-15,19$ \\
\hline Bélgica & 1608 & 671,43 & 0,37 & 322,18 & 8,91 & 70,83 & 35,14 & 92,72 & 1365 & 659,65 & 84,89 & $-1,53$ \\
\hline Taiwán & 1454 & 489,55 & 0,26 & 249,55 & 10,12 & 63,69 & 19,81 & $-25,90$ & 1308 & 540,00 & 89,96 & 8,56 \\
\hline Hong Kong & 1333 & 391,46 & 0,49 & 177,88 & 8,95 & 73,44 & 38,33 & 94,43 & 1074 & 327,03 & 80,57 & $-13,11$ \\
\hline Finlandia & 1327 & 692,31 & 0,49 & 370,55 & 9,76 & 74,53 & 31,73 & 89,32 & 1127 & 596,72 & 84,93 & $-12,06$ \\
\hline Israel & 1230 & 307,37 & 0,42 & 184,63 & 10,65 & 76,10 & 23,98 & 96,38 & 1083 & 291,86 & 88,05 & $-B, 81$ \\
\hline Sudáfrica & 1207 & 964,29 & 0,51 & 243,91 & 4,51 & 61,72 & 23,53 & $-3,69$ & 1087 & 962,16 & 90,06 & $-0,20$ \\
\hline Malasia & 1191 & 3340,00 & 0,43 & 237,02 & 2,72 & 56,00 & 23,93 & $-41,86$ & 1084 & 3157,14 & 91,02 & $-5,32$ \\
\hline Dinamarca & 1182 & 823,91 & 0,36 & 310,44 & 8,47 & 72,59 & 32,57 & 7,46 & 979 & 854,05 & 82,83 & 3,26 \\
\hline Nueva Zelanda & 1119 & 169,91 & 0,56 & 37,69 & 7,62 & 68,72 & 29,58 & 45,35 & 966 & 148,54 & 86,33 & $-7,92$ \\
\hline Suiza & 1033 & 952,78 & 0,18 & 444,37 & 9,98 & 71,25 & 46,85 & 37,73 & 787 & 935,71 & 76,19 & $-1,62$ \\
\hline Singapur & 999 & $\square 641,51$ & 0,39 & 234,96 & 10,16 & 78,78 & 46,95 & 61,08 & 761 & $\square 534,88$ & 76,18 & $-14,38$ \\
\hline Noruega & 914 & $\square 77,06$ & 0,34 & 251,64 & 8,88 & 74,51 & 31,18 & 41,67 & 766 & 713,79 & 83,81 & $B \beta, 93$ \\
\hline
\end{tabular}


Tabla 2. Impacto normalizado, Excelencia10, \% Excelencia10, Excelencia liderada, \%Excelencia liderada, Excelencia1 y \% Excelencia 1 , de los 27 países más productivos y tasas de variación del trienio 2015-2017 con respecto al trienio 2003-2005.

\begin{tabular}{|c|c|c|c|c|c|c|c|c|c|c|c|c|c|c|c|}
\hline País & \begin{tabular}{|c|} 
Impacto \\
Normalizado
\end{tabular} & TV & \begin{tabular}{|c|} 
Excelencia \\
10
\end{tabular} & TV & $\begin{array}{c}\text { \% Excelencia } \\
10\end{array}$ & TV & $\begin{array}{c}\text { Excelencia } \\
10 \text { liderada }\end{array}$ & TV & $\begin{array}{c}\text { \% Excelencia } \\
10 \text { liderada }\end{array}$ & TV & $\begin{array}{c}\text { Excelencia } \\
1\end{array}$ & TV & \begin{tabular}{|r|} 
Excel \\
1
\end{tabular} & encia & TV \\
\hline Estados Unidos & 1,26 & $-0,25$ & $\mid 6158$ & 184,58 & 14,08 & 5,05 & $|5563|$ & 167,76 & 12,72 & $-1,16$ & 653 & 174,24 & & 1,49 & 1,23 \\
\hline Reino Unido & 1,28 & 12,59 & 1539 & 266,94 & 12,90 & 11,85 & 1246 & 205,31 & 10,45 & $-6,94$ & 162 & 253,85 & & 1,36 & 7,86 \\
\hline España & $0,66[$ & $-27,62$ & 440 & 1283,33 & 6,74 & $-33,66$ & 338 & 1027,27 & 5,17 & $-45,94$ & 37 & 100,00 & $\square$ & 0,57 & 100,00 \\
\hline Australia & 1,11 & 6,14 & 632 & 561,11 & 12,04 & 20,31 & 503 & 551,72 & 9,58 & 18,60 & 56 & 433,33 & & 1,07 & $-2,94$ \\
\hline China & 0,76 & 106,94 & 290 & 12400,00 & 5,67 & 497,55 & 212 & 9200,00 & 4,14 & 344,58 & 29 & 100,00 & $\square$ & 0,57 & 00,00 \\
\hline India & 0,81 & 2,14 & 197 & 2140,00 & 4,36 & $-13,09$ & 173 & 2375,00 & 3,83 & $-3,98$ & 12 & 100,00 & & 0,27 & 100,00 \\
\hline Alemania & 1,53 & 33,83 & 632 & 904,55 & 15,52 & 47,07 & 496 & 709,09 & 12,18 & 18,46 & 70 & \#\#\#\#曲 & & 1,72 & 236,76 \\
\hline Canadá & 1,21 & 3,13 & 475 & 276,32 & 12,23 & $-15,50$ & 358 & 278,57 & 9,22 & $-14,99$ & 50 & 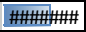 & & 1,29 & 214,40 \\
\hline Brasil & 0,33 & $-2,79$ & 55 & 100,00 & 1,69 & 100,00 & 32 & 100,00 & 0,98 & 100,00 & 8 & 100,00 & & 0,25 & 100,00 \\
\hline Países Bajos & 1,85 & 15,28 & 743 & 448,84 & 23,34 & 61,57 & 587 & 378,38 & 18,44 & 40,83 & 85 & \#\#\#\#\#\# & & 2,67 & 23,83 \\
\hline Francia & 0,76 & 191,57 & 181 & $\square 1500,00$ & 6,69 & 322,67 & 121 & 800,00 & 4,47 & 137,76 & 23 & 100,00 & $=$ & 0,85 & 100,00 \\
\hline Corea del Sur & 1,23 & $-27,74$ & 296 & 633,33 & 12,95 & $-18,97$ & 221 & 911,11 & 9,67 & 11,72 & 39 & 425,00 & & 1,71 & 41,99 \\
\hline Italia & 1,16 & 6,74 & 233 & 791,67 & 11,07 & $-2,42$ & 166 & 581,82 & 7,89 & $-25,39$ & 23 & 800,00 & & 1,09 & $-1,51$ \\
\hline Japón & 0,85 & 8,66 & 115 & 227,27 & 6,07 & $\mid-33,80$ & 74 & 200,00 & 3,90 & $-39,31$ & 13 & 400,00 & $\square$ & 0,69 & 1,14 \\
\hline Suecia & 1,75 & $-22,26$ & 291 & 723,08 & 17,06 & 8,19 & 222 & 650,00 & 13,01 & $-1,41$ & 29 & 800,00 & & 1,70 & 18,30 \\
\hline Bélgica & 1,27 & 18,87 & 250 & 880,00 & 15,55 & 27,04 & 196 & 688,89 & 12,19 & 2,26 & 23 & 300,00 & & 1,43 & $-48,15$ \\
\hline Taiwán & $0,91[$ & $-42,55$ & 215 & 490,91 & 14,79 & 0,23 & 190 & 728,57 & 13,07 & 40,54 & 18 & 500,00 & & 1,24 & 1,78 \\
\hline Hong Kong & 1,23 & 6,40 & 182 & 570,00 & 13,65 & 36,33 & 136 & 411,11 & 10,20 & 4,00 & 15 & 100,00 & & 1,13 & 100,00 \\
\hline Finlandia & 1,53 & 20,14 & 218 & 1011,11 & 16,43 & 40,24 & 172 & 900,00 & 12,96 & 26,21 & 21 & 600,00 & & 1,58 & 11,65 \\
\hline \begin{tabular}{|l|} 
Israel \\
\end{tabular} & 1,45 & 20,95 & 197 & 321,43 & 16,02 & 3,45 & 160 & 333,33 & 13,01 & 6,37 & 15 & 100,00 & & 1,22 & 00,00 \\
\hline Sudáfrica & $0,62[$ & $-38,02$ & 72 & 580,00 & 5,97 & $-36,11$ & 51 & 450,00 & 4,23 & $-48,32$ & 5 & 100,00 & ] & 0,41 & 81,21 \\
\hline Malasia & 0,71 & 40,66 & 66 & 100,00 & 5,54 & 100,00 & 43 & 100,00 & 3,61 & 100,00 & 12 & 100,00 & & 1,01 & 00,00 \\
\hline Dinamarca & 1,52 & 54,74 & 212 & 2500,00 & 17,94 & 181,41 & 166 & 2900,00 & 14,04 & 224,71 & 21 & 100,00 & & 1,78 & 00,00 \\
\hline Nueva Zelanda & 0,79 & 7,21 & 95 & 91,67 & 8,49 & $-28,99$ & 70 & 100,00 & 6,26 & $-25,90$ & 5 & $\mid 100,00$ & & 0,45 & 400,00 \\
\hline \begin{tabular}{|l|} 
Suiza \\
\end{tabular} & 1,68 & $-8,76$ & 196 & $\mid 1150,00$ & 18,97 & 18,73 & 139 & 766,67 & 13,46 & $-17,68$ & 23 & 600,00 & & 2,23 & 33,51 \\
\hline Singapur & 1,82 & 37,31 & 178 & 788,89 & 17,82 & 19,88 & 127 & 783,33 & 12,71 & 19,13 & 24 & 800,00 & & 2,40 & 21,37 \\
\hline Noruega & 1,56 & 127,31 & 182 & 6800,00 & 19,91 & 714,58 & 144 & 5300,00 & 15,75 & 537,49 & 18 & 100,00 & & 1,97 & 100,00 \\
\hline
\end{tabular}

internacional (y con todos los indicadores de rendimiento independientes del tamaño). Aunque sí tiene correlación con otros indicadores su tasa de variación, de la que las más llamativas son con la TV de Ndoc y con la TV del \%Ndoc, lo que podría venir a corroborar lo indicado anteriormente, que los grandes crecimientos se pudieran deber a crecimientos de producción propia sin colaboración internacional. Los países con mayor porcentaje de liderazgo son India, Brasil y Estados Unidos. Los de menor son Singapur y Suiza. Contrariamente a lo que pasa con la tasa de variación de la colaboración internacional, lo cual es consistente con su correlación negativa, los países que aumentan el porcentaje de liderazgo son los que más han crecido en esta década. Mientras que los que más lo disminuyen son Francia, Holanda y Suecia.

El Impacto normalizado es un indicador de rendimiento independiente del tamaño y, por tanto, tendrá una alta correlación con el resto de indicadores de rendimiento independientes del tamaño. También tiene una correlación importante con la colaboración internacional. Los trabajos con colaboración internacional tienen una mayor citación entre otras razones porque la comunidad participante es mayor (Lancho-Barrantes et al., 2012; Guerrero-Bote; Olmeda-Gómez; De-Moya-Anegón, 2013). Los países que incrementan su impacto normalizado más del $100 \%$ son Francia, Noruega y China (muy por encima del crecimiento medio del $20,4 \%$ de los 27 países). El hecho de que Francia haya sido el país que ha aumentado más la colaboración internacional ha podido ser la causa de su aumento en citación. En el extremo opuesto, con tasa de variación negativa, cabría destacar los casos de Taiwán, Sudáfrica, Corea del Sur, España y Suecia. También aunque menor, tienen tasa negativa Suiza y Brasil. Se observa que aunque Brasil y España han tenido un crecimiento notable de producción científica en Comunicación, el Impacto normalizado se encuentra por debajo de la media de los 27 y su tasa de variación es negativa (como se ha indicado), por lo que se puede afirmar que producen más, pero de menor impacto. Es posible también que se deba a la reciente incorporación de revistas a la base de datos, lo que supone la incorporación inmediata de nuevos documentos, pero de un menor impacto porque son revistas emergentes.

La Excelencia10 es un indicador dependiente del tamaño, y tendrá gran correlación con el Ndoc y el resto de indicadores dependientes del tamaño. No obstante, se aprecian cambios, como el caso de Holanda (que pasaría de la décima posición en Ndoc a la tercera en Excelencia10) o el caso de Alemania (de la séptima a la cuarta, junto a Australia).

En relación con la Tasa de variación, se observa que todos los países estudiados incrementan el número de excellent papers. Los países que más lo hacen son China (con un aumento excepcional), Noruega, Dinamarca e India, a bastante distancia del resto.

El \% Excelencia10 correlaciona mucho con el resto de indicadores de rendimiento independientes del tamaño; por ejemplo, tiene una correlación del 0,94 con el Impacto normalizado para los datos estudiados. Brasil tiene menos del $2 \%$, e India, Malasia, China y Sudáfrica están también por debajo del 6\%. Mientras por encima del 17\% están Países Bajos, Noruega, Suiza, Dinamarca y Singapur. Los países que más decrecen en el período son Sudáfrica, Japón y España. Los que más incrementan, Noruega, China y Francia, todos ellos por encima del 300\%. 


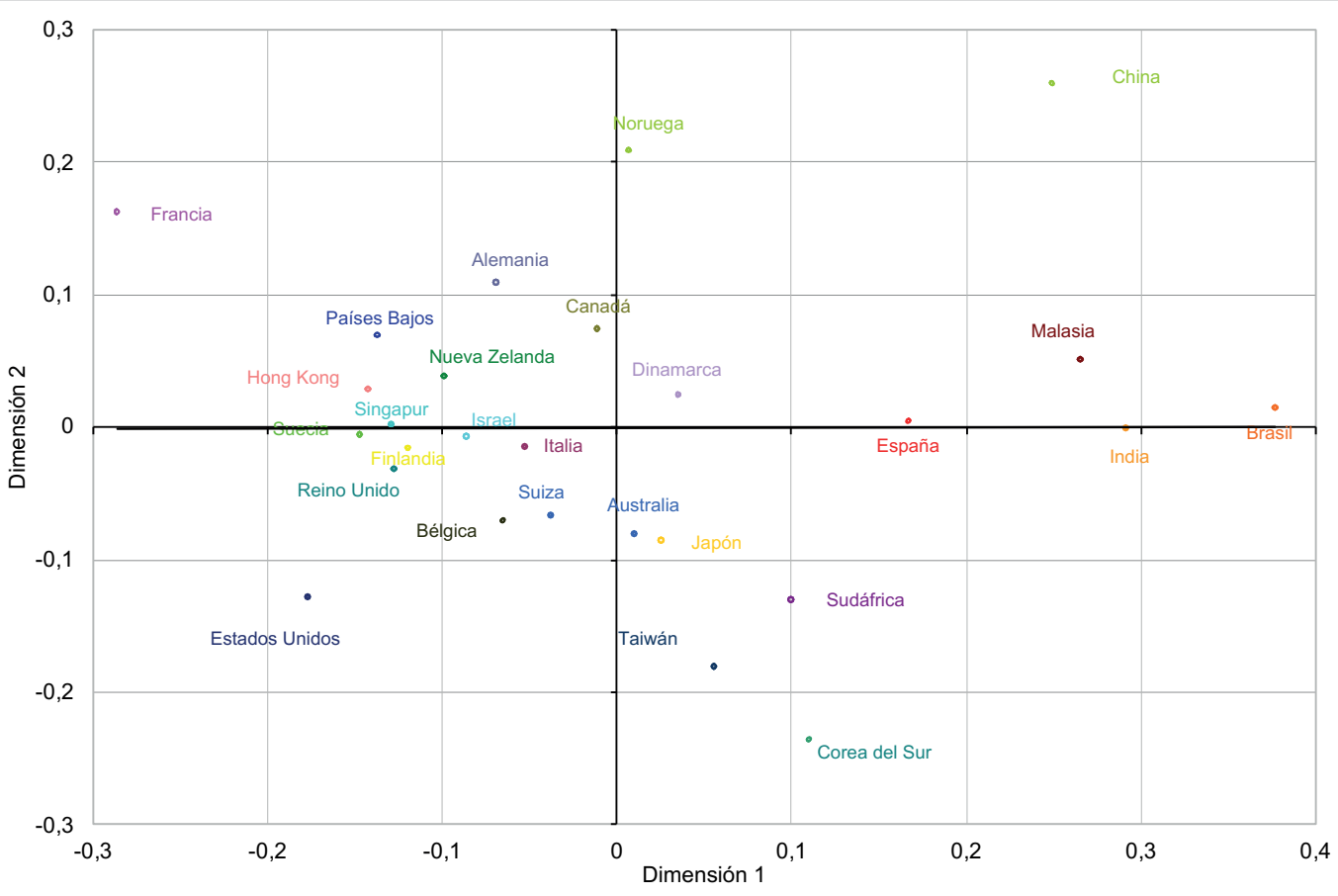

Figura 3. Resultado del escalamiento multidimensional de los países utilizando el procedimiento alscal, y las distancias euclídeas calculadas a partir de los indicadores de las tablas 2 y 3 , normalizados por el máximo valor y donde se ha reducido el peso de los indicadores independientes del tamaño al $10 \%$ (stress 0,07454).

Si de esa variables Excellencia10 nos quedamos con los trabajos liderados, los resultados son muy parecidos a los mencionados. Y algo semejante ocurre también con su tasa de variación; tanto Holanda como Alemania escalarían a los puestos tercero y quinto del ranking respectivamente.

En el \% Excelencia10 liderada se pueden observar los mismos rasgos que en el \% Excelencia 10; existe una correlación entre ambos indicadores de 0,99 . No obstante, mientras algunos pierden hasta el $42 \%$ de dicho porcentaje otros pierden el $10 \%$. El país que más pierde es Brasil, seguido de Japón y Malasia. En su variación, se observan rasgos muy parecidos a la variación del porcentaje de Excelencia10 (correlación de 0,97), aunque con diferencias, la reducción más grande vuelve a ser la de Sudáfrica, Japón y España.

El indicador Excelencia1 también tiene altas correlaciones con el resto de indicadores dependientes del tamaño, aunque ofrece otro cambio de posiciones. Por ejemplo, baja más China que se sitúa en sexta posición. Los primeros países son Estados Unidos, Reino Unido y Países Bajos. Los últimos Sudáfrica, Nueva Zelanda y Brasil. Los que más incrementan esta producción de Excelencia1 son Alemania, Canadá y Países Bajos con más del 1.000\%.

Algo similar se puede observar en el \% Excelencia1 con respecto a los indicadores de rendimiento independientes del tamaño. Los países con mayor \% Excelencia1 son Países Bajos, Singapur y Suiza, y los que menos Brasil e India. Los que más incrementan este \% Excelencia1 son Alemania, Países Bajos y Canadá con más de un 200\% en la tasa de variación. Los que más reducen su porcentaje de producción de Excelencia1 son Sudáfrica, Bélgica, Corea del Sur y Suiza, todos ellos por encima del $30 \%$.

Una visión más general del comportamiento de cada país se puede ver en el escalamiento multidimensional de la figura 3 (descrita metodológicamente en el apartado de metodología).

La dimensión 1 tiene una alta correlación con la tasa de variación de la producción $(0,88)$ y con la tasa de variación de la producción liderada y una correlación negativa con los indicadores de impacto. Es decir, en este mapa aparecen a la derecha los países que han incrementado su producción, y a la izquierda aquellos que tienen mayor impacto.

La mayor correlación de la dimensión 2 es con la tasa de variación del impacto normalizado $(0,75)$ y por tanto, con las tasas de variación de los indicadores de rendimiento independientes del tamaño. De modo que aparecen en la zona alta de la tabla los países que han incrementado la calidad de sus trabajos en el período.

Tal y como hemos realizado el mapa, es lógico que el tamaño no tenga una gran correlación con ninguna de las componentes, puesto que es lo que hemos tratado de evitar. Pero resulta llamativo que sean las tasas de variación, tanto de los indicadores de rendimiento independientes del tamaño, como de la producción las que mejor caractericen el mapa. La colaboración internacional, que es el indicador más independiente del resto, correlaciona negativamente con la primera coordenada -0,45. Los indicadores de rendimiento independientes del tamaño también vienen a tener una correlación negativa en torno a 0,5 con la primera dimensión (recordemos que con este tamaño de muestra, las correlaciones comienzan a ser significativas estadísticamente a partir de 0,374 al $5 \%$ y de 0,479 al $1 \%$ ). 


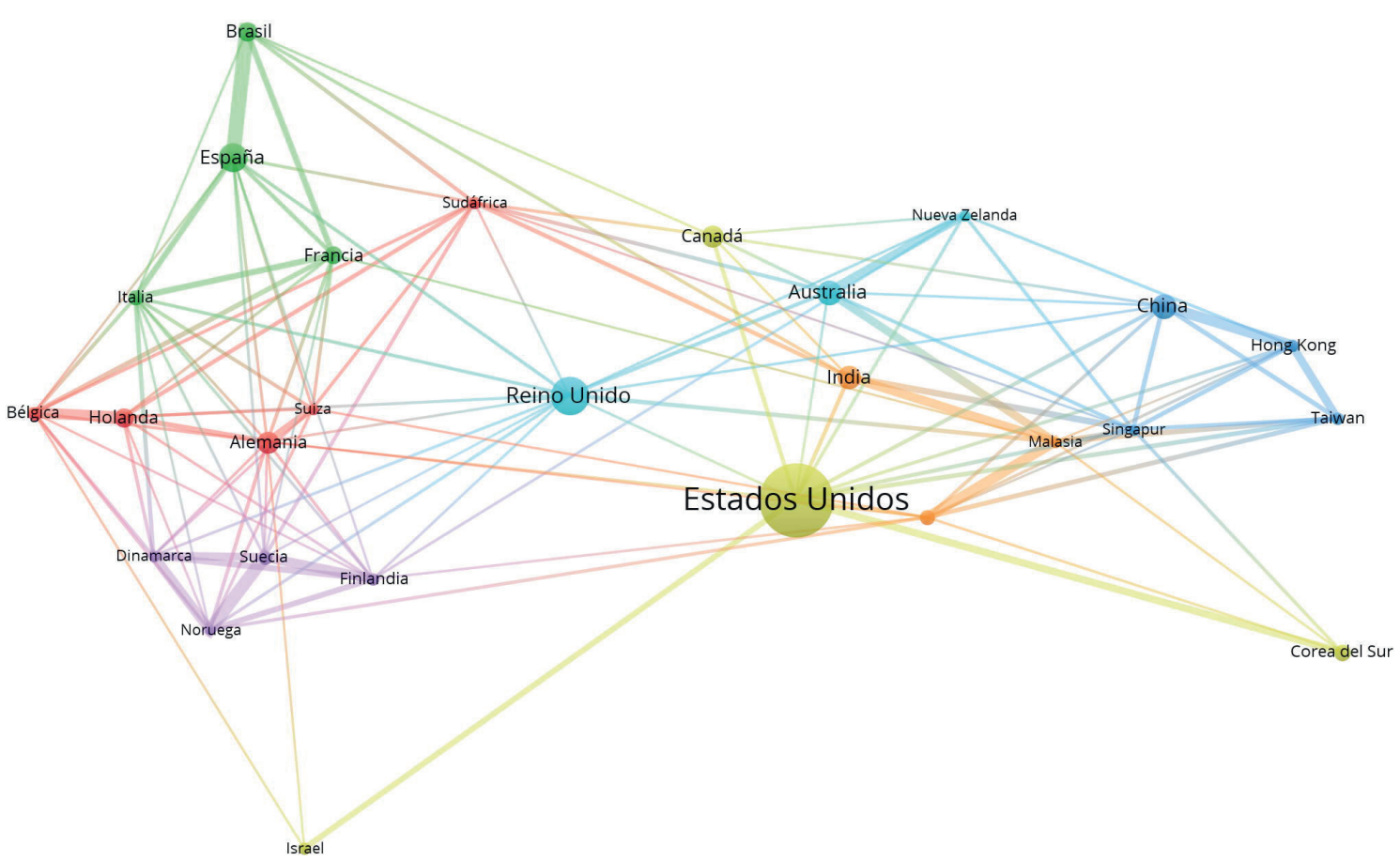

Figura 4. Red de colaboración científica entre los 27 países. Realizado con VOSviewer. El color corresponde a cada cluster.

En la figura 4 se muestra la red de colaboración entre estos 27 países. Se ha utilizado VOSviewer (VanEck; Waltman, 2010; Waltman; Van-Eck; Noyons, 2010) para hacer el mapa y el clustering (marcado con colores). Para ello se ha utilizado como peso del enlace el cociente de la ratio de documentos que supone y la probabilidad calculada a partir del Ndoc de cada país; es decir, la ratio entre la colaboración real y la colaboración probable dados los tamaños. Este peso también se ha utilizado para el grosor de los enlaces. El tamaño de los nodos es proporcional al Ndoc. Como los enlaces los hemos normalizado previamente nosotros tal y como se ha indicado, en el VOSViewer no se ha aplicado normalización, en la layout se han dejado como parámetros 2 en attraction y 1 en repulsion; y en clustering 0,80 en repulsion, 3 en Min. cluster size y se ha dejado marcado Merge small clusters. En la figura se han mostrado los enlaces con peso superior a 0,5 , porque los enlaces que representan una colaboración mayor de la probable son muy pocos.

En la figura 4 se pueden observar varios clusters. Si Estados Unidos es el país de mayor producción mundial, se puede ver que el algoritmo lo coloca en un cluster con Corea del Sur, Israel y Canadá, porque Estados Unidos es el país con el que más colaboran estos tres países. Sin embargo, de todas sus relaciones, sólo tienen un peso superior a uno (es decir, la colaboración supera lo probable por tamaño) con Corea del Sur, Israel y Taiwán. El algorimo lo coloca en una posición muy centrada porque tiene colaboraciones con muchos países.

Tenemos dos clusters asiáticos. Uno está formado por China, Hong Kong, Taiwán y Singapur. Estos cuatro países forman una comunidad muy relevan-
Tabla 3. Enlaces de colaboración internacional que son superiores a lo probable por tamaño

\begin{tabular}{|l|l|c|c|c|}
\hline \multicolumn{1}{|c|}{ País } & \multicolumn{1}{c|}{ País } & Ndoc & IN & Peso \\
\hline España & Brasil & 121 & 1,01 & 3,37 \\
\hline Suecia & Noruega & 52 & 3,02 & 2,93 \\
\hline Países Bajos & Bélgica & 181 & 1,78 & 2,47 \\
\hline China & Hong Kong & 178 & 1,24 & 2,43 \\
\hline Japón & Malasia & 20 & 0,94 & 2,36 \\
\hline Dinamarca & Noruega & 31 & 2,77 & 2,24 \\
\hline Suecia & Finlandia & 51 & 1,80 & 2,18 \\
\hline Australia & Nueva Zelanda & 74 & 0,84 & 2,03 \\
\hline India & Malasia & 11 & 1,64 & 2,02 \\
\hline Australia & Malasia & 39 & 0,79 & 1,87 \\
\hline Suecia & Dinamarca & 41 & 3,19 & 1,86 \\
\hline Taiwán & Hong Kong & 28 & 0,93 & 1,84 \\
\hline Alemania & Suiza & 133 & 1,90 & 1,67 \\
\hline Estados Unidos & Corea del Sur & 631 & 1,84 & 1,64 \\
\hline India & Singapur & 21 & 1,23 & 1,47 \\
\hline Finlandia & Dinamarca & 26 & 2,67 & 1,43 \\
\hline Brasil & Francia & 42 & 0,57 & 1,37 \\
\hline Francia & Italia & 88 & 1,85 & 1,37 \\
\hline Estados Unidos & Israel & 204 & 2,14 & 1,31 \\
\hline España & Italia & 98 & 1,62 & 1,30 \\
\hline Finlandia & Noruega & 19 & 1,84 & 1,30 \\
\hline Alemania & Países Bajos & 230 & 2,37 & 1,22 \\
\hline Taiwán & Malasia & 6 & 0,84 & 1,17 \\
\hline Francia & Bélgica & 55 & 0,86 & 1,12 \\
\hline Japón & Taiwán & 14 & 0,98 & 1,08 \\
\hline China & Singapur & 69 & 1,58 & 1,06 \\
\hline China & Taiwán & 40 & 1,59 & 1,06 \\
\hline Estados Unidos & Taiwán & 145 & 1,75 & 1,03 \\
\hline
\end{tabular}


te en términos de producción y sinergias colaborativas. La otra comunidad que destaca en Asia es la conformada por India, Malasia y Japón. A diferencia del cluster de Estados Unidos, en estos dos clusters todos los nodos están unidos con enlaces de peso superior a uno. No sólo eso sino que mantienen vinculos superiores.

Como segundo productor científico mundial en Comunicación, Reino Unido tiene otra posición central y forma otro cluster con países de la Commonwealth como son Australia y Nueva Zelanda. La posición central es lógicamente porque por su tamaño tiene colaboraciones con muchos países igual que Estados Unidos. Sin embargo, de todos esos enlaces de colaboración ninguno supera la unidad; es decir, no tiene ninguna colaboración superior a lo probable por tamaño. El único enlace con peso superior a la unidad en su cluster es entre Australia y Nueva Zelanda; Australia tiene un enlace con Malasia de 1,87.

Existe un cluster de países nórdicos formado por Dinamarca, Suecia, Finlandia y Noruega. Su característica principal es la gran colaboración existente entre ellos, con un enlace superior a la unidad entre todos los países, y ningún enlace superior a la unidad con ningún otro país. Además, son todos enlaces de muy alto impacto. Si nos fijamos en la tabla 3 , que muestra los enlaces de peso superior a la unidad, los cuatro enlaces de mayor impacto están en el cluster nórdico.

Existe otro cluster formado por España, Brasil, Italia y Francia. En este caso destaca la gran colaboración trasatlántica entre España y Brasil que da lugar al enlace de mayor peso. Y otro cluster europeo, formado por Alemania, Suiza, Holanda y Bélgica donde se ha incoporado Sudáfrica (aunque esta última sin ningún enlace de peso superior a la unidad). Estos dos clusters europeos están unidos entre sí mediante un enlace de peso superior a 1 entre Bélgica y Francia.

Si echamos un vistazo general a la red de la figura 4, podemos ver una red europea bastante densa y de gran impacto, y otra red de menor densidad e impacto de países del Pacífico (Asía+Oceanía). Y algunos países intermediarios sin grandes enlaces de colaboración como son Estados Unidos y Reino Unido.

Por otro lado, respecto a los impactos normalizados de los enlaces de peso superior a la unidad que se muestran en la tabla 3, podemos decir que en los enlaces de mayor impacto suele intervenir al menos un país europeo o Estados Unidos. Como excepción está Francia que interviene en dos enlaces con impacto inferior a la unidad. Los enlaces entre países asiáticos tienen bajo impacto.

\section{Conclusiones}

La producción en Comunicación ha crecido a ritmo muy elevado entre 2003 y 2018, muy superior al resto de la producción científica mundial. Parte de este crecimiento se debe al incremento de la cobertura de la base de datos Scopus (que ha aumentado en todas las disciplinas científicas) pero a su vez también puede ser consecuencia del crecimiento de la disciplina. El motivo principal

\section{El crecimiento medido a través de estas bases de datos puede estar reflejando más el cambio de hábitos de publicación de los académicos que su aumento de la actividad investigadora}

no es sólo el crecimiento natural de la comunidad académica. También ha sido determinante un cierto cambio de cultura académica que ha hecho que los resultados de investigación se internacionalicen a través de canales de comunicación presentes con más frecuencia en las fuentes de información internacionales. Este cambio de cultura académica puede venir forzado por las políticas públicas que incentivan de diferentes modos en los países la publicación en revistas indexadas en las bases de datos de referencia. Este hecho permite concluir que el crecimiento medido a través de esas bases de datos puede estar reflejando más el cambio de hábitos de publicación de los académicos que su aumento de la actividad investigadora, aunque resulta difícil discriminar en qué medida una u otra causa explican el crecimiento.

Los tres países de mayor producción son Estados Unidos, Reino Unido y España. Destaca el crecimiento de la producción de Malasia, Brasil, India, China y España. Fernández-Quijada y Masip (2013) informaron que España era el cuarto país europeo por detrás de Reino Unido, Países Bajos y Alemania (base de datos WoS y período 1980-2010). Aproximadamente la mitad del crecimiento español también es consecuencia de las revistas españolas incluidas en Scopus en el período. En 2013 el 78\% de los documentos españoles se publicaban en revistas españolas, sin embargo, desde entonces ese porcentaje ha ido decreciendo hasta llegar a cerca del 50\%. Además, también se está incrementando el porcentaje de trabajos que se publican en revistas españolas, pero no se publican en castellano sino directamente en inglés.

Los países que mayor impacto tienen son Países Bajos, Singapur, Suecia, Suiza, Noruega y Finlandia. De ellos destaca la evolución positiva de Noruega en todos los indicadores de impacto.

La colaboración internacional es menor que en otras disciplinas como por ejemplo Ciencias de los Alimentos (Guerrero-Bote; De-Moya-Anegón, 2015), lo que lleva a altos porcentajes de liderazgo en todos los países y no grandes variaciones entre los resultados totales y los resultados de la producción liderada. Sin embargo, hay una correlación significativa entre los indicadores de impacto independientes del tamaño, y el incremento en la tasa de colaboración científica, en torno a 0,6. Esta correlación pone de manifiesto un efecto común en muchas disciplinas: la relación causal existente entre colaboración internacional e impacto científico.

Existe una red de colaboración europea bastante densa y de gran impacto, y otra red de menor densidad e impacto de países del Pacífico (Asia y Oceanía). Y algunos países intermediarios sin grandes enlaces de colaboración como son Esta- 
dos Unidos y Reino Unido. Los tres países que no tienen enlaces con más colaboración de lo probable son precisamente Reino Unido, Sudáfrica y Canadá.

Por todo ello, quizá podemos decir que se trata de una disciplina emergente, que al estar entre la ciencia y la técnica quizá le faltan todavía referentes científicos comunes al conjunto de la disciplina y una estructura de colaboración científica más global. Quizá el carácter local de algunas temáticas influya en las dificultades para construir colaboraciones transnacionales de largo alcance. En sentido contrario la tecnificación de los procesos de comunicación está contribuyendo a que aparezcan visiones y análisis más globales en los trabajos.

\section{Referencias}

Álvarez-Nobell, Alejandro; Castillo-Esparcia, Antonio (2015). "Autores en revistas latinoamericanas de mayor impacto de comunicación". Opción, v. 31, n. 3, pp. 70-90.

https://www.redalyc.org/articulo.oa?id=31045567005

Archambault, Éric; Campbell, David; Gingras, Yves; Larivière, Vincent (2009). "Comparing bibliometric statistics obtained from the Web of Science and Scopus". Journal of the American Society for Information Science and Technology (Jasist), v. 60, n. 7, pp. 1320-1326.

https://doi.org/10.1002/asi.21062

Baladrón-Pazos, Antonio-José; Manchado-Pérez, Benjamín; Correyero-Ruiz, Beatriz (2017). “Estudio bibliométrico sobre la investigación en publicidad en España: temáticas, investigadores, redes y centros de producción (1980-2015)". Revista española de documentación científica, v. 40, n. 2, e170.

http://doi.org/10.3989/redc.2017.2.1411

Barnett, George A.; Huh, Catherine; Kim, Youngju; Park, Han-Woo (2011). "Citations among communication journals and other disciplines: a network analysis". Scientometrics, n. 88, pp. 449-469.

https://doi.org/10.1007/s11192-011-0381-2

Bornmann, Lutz; De-Moya-Anegón, Félix; Leydesdorff, Loet (2012). "The new excellence indicator in the World Report of the SCImago Institutions Rankings 2011". Journal of informetrics, v. 6, n. 2, pp. 333-335.

https://doi.org/10.1016/j.joi.2011.11.006

Castillo-Esparcia, Antonio; Carretón-Ballester, María-Carmen (2010). “Investigación en Comunicación. Estudio bibliométrico de las revistas de Comunicación en España". Comunicación y sociedad, v. 23, n. 2, pp. 289-327.

https://revistas.unav.edu/index.php/communication-and-society/article/view/36234

https://doi.org/10.15581/003.23.2.289-327

Castillo-Esparcia, Antonio; Rubio-Moraga, Ángel; Almansa-Martínez, Ana (2012). "La investigación en Comunicación. Análisis bibliométrico de las revistas de mayor impacto del ISI". Revista latina de comunicación social, v. 67, pp. 248-270. https://doi.org/10.4185/RLCS-067-955-248-270

Castillo-Esparcia, Antonio; Xifra, Jordi (2006). "Investigación bibliométrica de las tesis doctorales españolas sobre relaciones públicas (1965-2005)". Anàlisi: quaderns de comunicació i cultura, n. 34, pp. 141-161.

https://www.raco.cat/index.php/Analisi/article/view/55449

Corera-Álvarez, Elena; De-Moya-Anegón, Félix (2009). “Chemistry in Spain: bibliometric analysis through Scopus”. Chemistry today, v. 27, n. 6, pp. 61-64.

De-Filippo, Daniela (2013). "La producción científica española en comunicación en WoS. Las revistas indexadas en SSCI (2007-12)". Comunicar, v. 21, n. 41, pp. 25-34.

http://doi.org/10.3916/C41-2013-02

De-las-Heras-Pedrosa, Carlos; Martel-Casado, Telva; Jambrino-Maldonado, Carmen (2018). “Análisis de las redes académicas y tendencias científicas de la comunicación en las universidades españolas". Revista prisma social, n. 22, pp. 229-246.

https://revistaprismasocial.es/article/view/2584/2760

De-Moya-Anegón, Félix; Chinchilla-Rodríguez, Zaida; Vargas-Quesada, Benjamín; Corera-Álvarez, Elena; Muñoz-Fernández, Francisco-José; González-Molina, Antonio; Herrero-Solana, Víctor (2007). "Coverage analysis of Scopus: A journal metric approach". Scientometrics, v. 73, pp. 53-78.

https://doi.org/10.1007/s11192-007-1681-4

De-Moya-Anegón, Félix; Guerrero-Bote, Vicente P.; Bornmann, Lutz; Moed, Henk F. (2013). "The research guarantors of scientific papers and the output counting: A promising new approach". Scientometrics, v. 97, pp. 421-434.

https://doi.org/10.1007/s11192-013-1046-0 
Demeter, Marton (2018). "Changing center and stagnant periphery in communication and media studies: National diversity of major international journals in the field of communication from 2013 to 2017". International journal of communication, v. 12, pp. 2893-2921.

https://ijoc.org/index.php/ijoc/article/view/9122/2402

Fernández-Quijada, David; Masip, Pere (2013). “Tres décadas de investigación española en comunicación: hacia la mayoría de edad". Comunicar, v. 21, n. 41, 1-24.

https://doi.org/10.3916/C41-2013-01

Fernández-Quijada, David; Masip, Pere; Bergillos, Ignacio (2013). “El precio de la internacionalidad: la dualidad en los patrones de publicación de los investigadores españoles en comunicación". Revista española de documentación científica, v. 36, n. 2, e010.

https://doi.org/10.3989/redc.2013.2.936

Galán-Arribas, Rafael; Herrero-Gutiérrez, Francisco-Javier; Vergara-Fragoso, Martha; Martínez-Arcos, Cruz-Alberto (2018): "Estudios sobre el podcast radiofónico: revisión sistemática bibliográfica en WoS y Scopus que denota una escasa producción científica". Revista latina de comunicación social, n. 73, pp. 1398-1411.

https://doi.org/10.4185/RLCS-2018-1313

Gorraiz, Juan; Gumpenberger, Christian; Wieland, Martin (2011). “Galton 2011 revisited: a bibliometric journey in the footprints of a universal genius". Scientometrics, v. 88, n. 2, pp. 627-652.

https://doi.org/10.1007/s11192-011-0393-y

Guerrero-Bote, Vicente P.; De-Moya-Anegón, Félix (2015). “Analysis of scientific production in food science from 2003 to 2013". Journal of food science, v. 80, n. 12, pp. R2619-R2626.

http://doi.org/10.1111/1750-3841.13108

Guerrero-Bote, Vicente P.; Olmeda-Gómez, Carlos; De-Moya-Anegón, Félix (2013). “Quantifying the benefits of international scientific collaboration". Journal of the American Society for Information Science and Technology, v. 64, n. 2, pp. 392-404.

https://doi.org/10.1002/asi.22754

Hane, Paula J. (2004). “Elsevier announces Scopus service”. Information today, March 15.

http://newsbreaks.infotoday.com/nbreader.asp?ArticlelD $=16494$

Jacsó, Péter (2011). "The h-index, h-core citation rate and the bibliometric profile of the Scopus database". Online information review, v. 35, n. 3, pp. 492-501.

https://doi.org/10.1108/14684521111151487

Kolle, Shankar-Reddy; Rabindranath, Manukonda; Shankarappa, T. H. (2019). "Characteristics of highly cited articles in communication (1989 to 2018): A Web of Science-based analysis". Media watch, v. 10, n. 3, pp. 645-663.

https://doi.org/10.15655/mw/2019/v10i3/49700

Lancho-Barrantes, Bárbara S.; Guerrero-Bote, Vicente P.; Chinchilla-Rodríguez, Zaida; De-Moya-Anegón, Félix (2012). "Citation flows in the zones of influence of scientific collaborations". Journal of the American Society for Information Science and Technology, v. 63, n. 3, pp. 481-489.

https://doi.org/10.1002/asi.21682

Lauf, Edmund (2005). "National diversity of major international journals in the field of communication”. Journal of communication, v. 55, n. 1, pp. 139-151.

http://doi.org/10.1111/j.1460-2466.2005.tb02663.x

Leydesdorff, Loet; De-Moya-Anegón, Félix; Guerrero-Bote, Vicente P. (2010). "Journal maps on the basis of Scopus data: A comparison with the Journal Citation Reports of the ISI". Journal of the American Society for Information Science and Technology, v. 61, n. 2, pp. 352-369.

https://doi.org/10.1002/asi.21250

Leydesdorff, Loet; Probst, Carole (2009). "The delineation of an interdisciplinary specialty in terms of a journal set: The case of communication studies". Journal of the American Society for Information Science and Technology, v. 60, n. 8, pp. 1709-1718.

https://doi.org/10.1002/asi.21052

Méndez-Rátiva, Claudia-Patricia; Gregorio-Chaviano, Orlando (2014). “Aproximación a la comunicación desde la perspectiva teórica y bibliométrica. Un análisis en Web of Science 2008-2012". Signo y pensamiento, v. 33, n. 64, pp. 114-135. https://doi.org/10.11144/Javeriana.SyP33-64.actb

Montero-Díaz, Julio; Cobo, Manuel-Jesús; Gutiérrez-Salcedo, María; Segado-Boj, Francisco; Herrera-Viedma, Enrique (2018). “Mapeo científico de la Categoría 'Comunicación' en WoS (1980-2013)”. Comunicar, v. 26, n. 55, pp. 81-91.

https://doi.org/10.3916/C55-2018-08 
Moreno-Delgado, Alicia; Repiso, Rafael; Montero-Díaz, Julio (2020). “Análisis de redes sociales de la producción científica sobre programación televisiva”. Icono14, v. 18, n. 1, pp. 123-154.

https://doi.org/10.7195/ri14.v18i1.1484

Park, Han-Woo; Leydesdorff, Loet (2009). "Knowledge linkage structures in communication studies using citation analysis among communication journals". Scientometrics, v. 81, n. 1, pp. 157-175.

https://doi.org/10.1007/s11192-009-2119-y

Pickering, Bobby (2004). “Elsevier prepares Scopus to rival ISI Web of science”. Information world review, n. 200. http://140.234.252.185/c/articles/12723322/elsevier-prepares-scopus-rival-isi-web-science

Rehn, Catharina; Kronman, UIf (2008). Bibliometric handbook for Karolinska Institutet. Karolinska Institutet University Library. Version 1.05.

https://doi.org/10.13140/RG.2.1.1480.9447

Repiso, Rafael; Torres-Salinas, Daniel; Delgado-López-Cózar, Emilio (2011a). "Análisis de la investigación sobre radio en España: una aproximación a través del análisis bibliométrico y de redes sociales de las tesis doctorales defendidas en España entre 1976-2008". Estudios sobre el mensaje periodístico, v. 17, n. 2, pp. 417-429.

https://doi.org/10.5209/rev_ESMP.2011.v17.n2.38123

Repiso, Rafael; Torres-Salinas, Daniel; Delgado-López-Cózar, Emilio (2011b). “Análisis bibliométrico y de redes sociales en tesis doctorales españolas sobre televisión (1976/2007)". Comunicar, v. 37, pp. 151-159.

https://doi.org/10.3916/C37-2011-03-07

Rodríguez-Gómez, Eduardo-Francisco; Goyanes, Manuel; Rosique-Cedillo, Gloria (2018). “La investigación en comunicación en España: temporalidad laboral, producción intensiva y competitividad". Communication \& society, v. 31, n. 4, pp. 229-242.

https://revistas.unav.edu/index.php/communication-and-society/article/view/35684

Romo-Fernández, Luz M.; López-Pujalte, Cristina; Guerrero-Bote, Vicente P.; De-Moya-Anegón, Félix (2011). “Analysis of Europe's scientific production on renewable energies". Renewable energy, v. 36, n. 9, pp. 2529-2537.

https://doi.org/10.1016/j.renene.2011.02.001

SCImago (2020a). SJ\&CR. SCImago Journal \& Country Rank.

http://www.scimagojr.com

SCImago (2020b). SIR. SCImago Institutions Rankings.

http://www.scimagoir.com

Van-Eck, Nees-Jan; Waltman, Ludo (2010). "Software survey: VOSviewer, a computer program for bibliometric mapping". Scientometrics, v. 84, n. 2, pp. 523-538.

https://doi.org/10.1007/s11192-009-0146-3

Waltman, Ludo; Van-Eck, Nees-Jan; Noyons, Ed C. M. (2010). "A unified approach to mapping and clustering of bibliometric networks". Journal of informetrics, v. 4, n. 4, pp. 629-35.

https://doi.org/10.1016/j.joi.2010.07.002

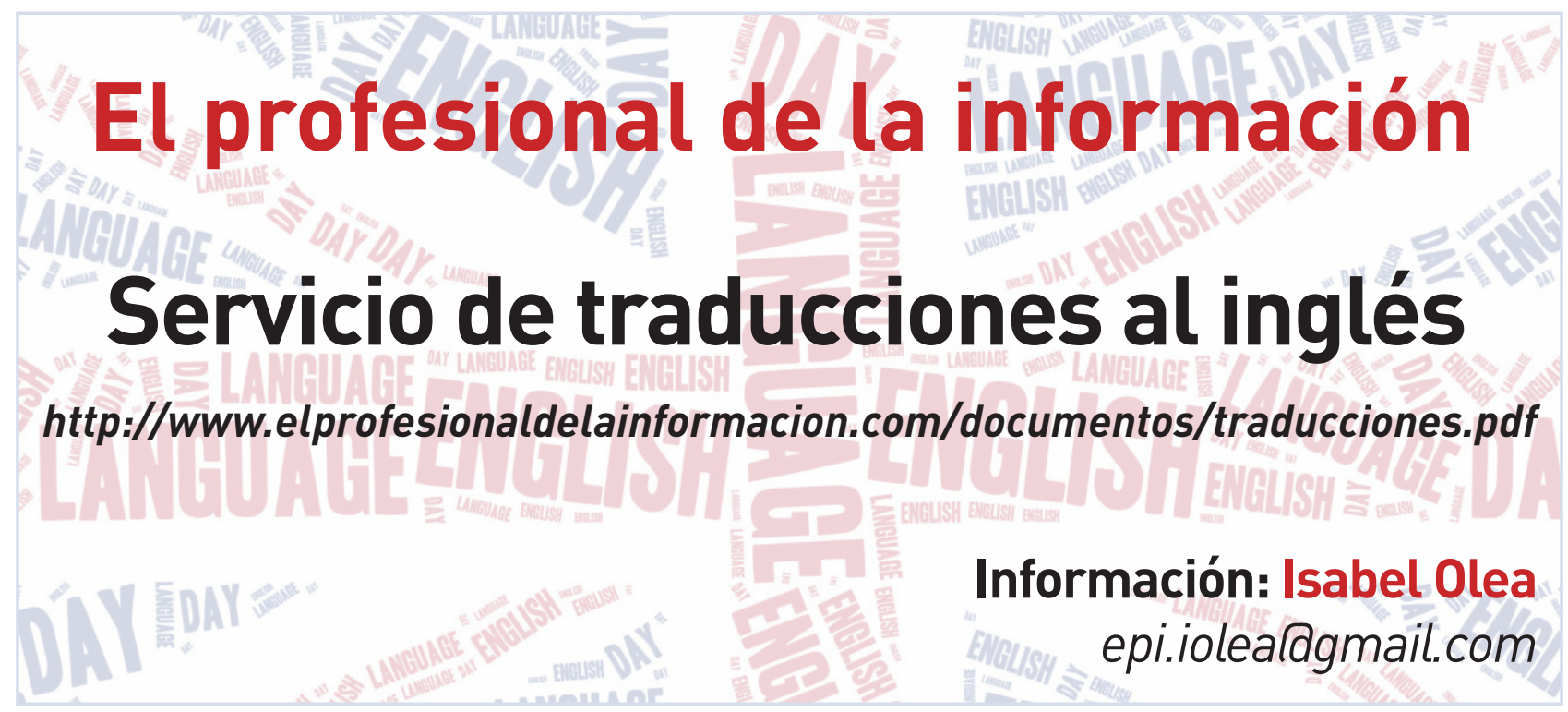

